\title{
NEBRIJA, AUTORIDAD EN EL TESORO DE COVARRUBIAS
}

\author{
Gloria Guerrero Ramos
}

Se ha dicho que las Introductiones nebrisenses devolvieron a la España bárbara los 'studia humanitatis', los únicos quehaceres dignos del hombre, y le abrieron el horizonte de una edad de oro, es decir, trajeron a la Península, en palabras de Francisco Rico, "el Renacimiento. Entiéndase (cuando menos): trajeron la modernidad a la lengua y la traza de la literatura" ${ }^{1}$. Efectivamente, las Introductiones fueron el punto de partida del humanismo, pero no sólo ellas supusieron la entrada del mismo en España, sino toda la obra de Elio Antonio de Nebrija, especialmente su Gramática y sus Diccionarios.

Nebrija no es sólo el primer gramático, sino que con el Vocabulario de romance en latín ${ }^{2}$ se convierte también en el primer lexicógrafo español. No olvidamos a Alfonso de Palencia, anterior a Nebrija, cuya obra Universal vocabulario en latín y en romance ${ }^{3}$ es un verdadero diccionario, pero parte del latín y se limita a traducir al español lo que expresaba en latín. No negamos su posible aportación a la obra lexicográfica de Nebrija, sin embargo, es todavía una obra de carácter netamente medieval ${ }^{\star}$; en cambio, la moder-

1 Francisco Rico, “Lección y herencia de Elio Antonio de Nebrija, 1481-1981", en Nebrija y la introducción del Renacimiento en España, Actas de la III Academia Literaria Renacentista, Universidad de Salamanca, 1983, págs. 9-14. Tomamos la cita de la pág. 9.

2 Elio Antonio de Nebrija, Vocabulario español-latino, Salamanca (¿1495?), facsímil de la Real Academia Española, Madrid, 1951; reeditado nuevamente en 1989. A partir de ahora citaremos Vocabulario.

3 Alfonso de Palencia, Universal vocabulario en latin y en romance, Sevilla (1490), facsímil, Comisión Permanente de la Asociación de Academias de la Lengua Española, Madrid, 2 vols., 1967.

- Annamaria Gallina, Contributi alla storia della lessicografia italo-spagnola dei secoli XVI e XVII, Florencia, Leo S. Olschki-Editore, 1959, pág. 13. 
nidad de Nebrija salta inmediatamente a la vista ${ }^{5}$. Tampoco es Nebrija el primero al que se le ocurre realizar un diccionario partiendo de una lengua viva. Se le adelantó el valenciano Juan Esteve, quien en 1489 publica un diccionario de frases y expresiones en catalán, con sus correspondencias en latín ${ }^{6}$, pero no se puede comparar con el de Nebrija, y "conviene, por contraste, insistir en la mayor utilidad y concepción moderna del Vocabulario nebrisense" ?

Bien es sabido que Nebrija se convirtió en el precedente de la lexicografía española posterior. Fue tomado como modelo por lexicógrafos extranjeros para la construcción de vocabularios bilingües o plurilingües, pero también fue utilizado por lexicógrafos españoles para la creación de diccionarios monolingües.

Hemos tenido ocasión de demostrar ${ }^{8}$ que Nebrija ha sido utilizado por Pedro Alcalá ${ }^{9}$, Cristóbal de las Casas ${ }^{10}$, Percyvall ${ }^{11}$, Minsheu ${ }^{12}$, Palet ${ }^{13}$, César Oudin ${ }^{14}$, Franciosini ${ }^{15}$ y Sobrino ${ }^{16}$ para la creación de sus dicciona-

5 Germán Colón y Amadeu-J. Soberanas, en el estudio preliminar del facsímil del Diccionario latino-español de Nebrija, Salamanca (1492), Barcelona, Puvill-Editor, 1979 , pág. 24.

6 Joan Esteve, Liber elegantiarum, Venecia, 1489. Manejamos el ejemplar existente en la Biblioteca de Catalunya con la signatura 11-VIII-4. También hay una reproducción facsimilar reciente, Castellón de la Plana, 1988.

7 Germán Colón y Amadeu-J. Soberanas, en el estudio citado, pág. 25, nota 35.

8 En nuestra tesis doctoral, El léxico en el Diccionario (1492) y en el Vocabulario ( $\left.{ }_{6} 14959\right)$ de Nebrija, editada en microfichas por el Servicio de Publicaciones de la Universidad de Málaga e impresa por E. T. D., S. A., Micropublicaciones en Barcelona, Málaga, 1988, págs. 290-528, microfichas 4-6.

9 Pedro de Alcalá, Vocabulista aravigo en letra castellana, Granada, 1505. Manejamos el ejemplar de la Biblioteca Nacional de Madrid, signatura R/2169. Hemos utilizado también la impresión microfotográfica realizada por The Hispanic Society of America, Nueva York, 1928. Y además la edición realizada por Paul de Lagarde, Gottingae (1883), 1971, que lleva por título: Petri Hispani lingua arabica libri duo.

10 Christoval de las Casas, Vocabulario de las dos lenguas toscana y castellana..., Sevilla, 1570. Manejamos el ejemplar de la Biblioteca Nacional de Madrid, signatura $\mathrm{R} / 24098$.

11 Richard Percyvall, Bibliotheca Hispanica. Containing a Grammar, with a Dictionaric in Spanish, English, and Latine, London, by Iohn Iackson, for Richard Watkins, 1591. Manejamos el ejemplar existente en la Biblioteca Nacional de Madrid, signatura $3 / 4954$.

12 Iohn Minshev, A dictionarie in spanish and english, London, 1599. Manejamos el ejemplar existente en la Biblioteca Nacional de Madrid, signatura R/45017.

18 Ioan Palet, Diccionario muy copioso de la lengua española y francesa, Paris, Matthieu Guillimet, 1604. Manejamos el ejemplar existente en la Biblioteca Nacional de Madrid, signatura $\mathrm{R} / 23641$.

14 César Oudin, Tesoro de las dos lenguas francesa y española, Paris, Marc Orry, 1607. Manejamos las microfichas realizadas por E. T. D., Colección Hispania Lingüis- 
rios. Sin embargo, son muy pocos los que tienen la honradez de Covarrubias al reconocer que se han servido de Nebrija. La obra lexicográfica del nebrisense gozó de suficiente popularidad para ser conocida de todos los lexicógrafos coetáneos y posteriores. Pedro Alcalá hace constar en el prólogo de su Vocabulista aravigo la deuda contraída con Nebrija, pero en este caso es lógico que lo hiciese, ya que la obra de Alcalá es, prácticamente, el diccionario de Nebrija traducido al árabe. Oudin menciona a Nebrija alguna vez, de la misma manera que menciona a Covarrubias al emplearlo en la segunda edición del Tesoro de las dos lenguas española y francesa (1616). En definitiva, Nebrija es conocido, estudiado, manejado y utilizado, aunque sin ser mencionado por parte de los autores de diccionarios.

Sorprende, pues, que Covarrubias cite explícitamente a Nebrija como autoridad, aunque no siempre, solamente cuando le conviene. Hemos elegido el diccionario de Covarrubias ${ }^{17}$ como representante de la lexicografía monolingüe española, porque supone el inicio de la misma y simboliza, perfectamente, todo lo que la lexicografía española debe a Nebrija.

La originalidad de la obra lexicográfica de Covarrubias, como la de cualquier autor, no niega en absoluto la presencia de influencias ajenas. Tan lógico es que todos los diccionarios sean, en parte, originales como el que los lexicógrafos se sirvan unos de otros, o bien utilicen como fuentes obras literarias.

En el caso de Covarrubias, sus fuentes literarias han sido más o menos estudiadas ${ }^{18}$. En cambio, Nebrija como fuente lexicográfica no ha sido es-

tica, Barcelona, 1985. También empleamos el ejemplar de la 2.a ed., París, 1616, existente en la Biblioteca Nacional de Madrid, signatura R-i/32.

15 Lorenzo Franciosini, Vocabulario español, e italiano aora nuevamente sacado a luz, Roma, A costa de Iuan Angel Rufineli y Angel Manni, 1620. Manejamos el ejemplar existente en la Biblioteca Nacional de Madrid, signatura 3/73623.

1 Francisco Sobrino, Diccionario nuevo de las lenguas española y francesa, Bruselas, Francisco Foppens, 1705. Manejamos el ejemplar existente en la Biblioteca Nacional de Madrid, signatura 3/42977.

17 Sebastián de Covarrubias Orozco, Tesoro de la lengua castellana o española, Madrid, Luis Sánchez, 1611. Manejamos el ejemplar R/14431 existente en la Biblioteca Nacional de Madrid, la edición preparada por Martín de Riquer, S. A., Horta, Barcelona, 1943, y la de Ediciones Turner, Madrid, 1977.

18 Véase José Romera Castillo, “Don Juan Manuel (El Conde Lucanor) autoridad en el Tesoro de Covarrubias", en Don Juan Manuel, VII Centenario, Universidad de Murcia, Academia Alfonso El Sabio, Murcia, 1982, págs. 313-324. Véase también José Romera Castillo, "Autoridades medievales del Tesoro de Covarrubias", en Anuario de Letras, XXII, 1984, págs. 251-260. Véase además Manuel Seco, "Un lexicógrafo de la generación de Cervantes (Notas sobre el Tesoro de Covarrubias)", en Instituto de Bachillerato Cervantes, Miscelanea en su cincuentenario, 1931-1981, Madrid, Ministerio de Educación y Ciencia, 1982, págs. 229-243. Véase, por último, especialmente Manuel Seco, 
tudiado, pese a que no se dude en reconocer que fue muy apreciado por Covarrubias en muchos aspectos ${ }^{19}$.

A Nebrija como autoridad lo encontramos citado a lo largo del Tesoro de la lengua castellana o española de Sebastián de Covarrubias en 241 entradas — en dos de ellas aparece citado dos veces-, que son las siguientes:

abucasta, achaque, açumbre, a̧utea, adelante, adesoras, adufre, adulçar, afán, afrechos, albañar, albarrán, albatoza, alcaçava, alcacel, alcaduz (en dos ocasiones), alcavala, alerzo, algorfa, alhombra, almadén, almadena, almadraque, almario, almea, almenara (en dos ocasiones), almeja, almirante, almires, almirón, almorrana, almoxarife, almud, alquitara, alquitira, alterar, alvar, alvarquoque, anca, anchova, anduares, antojo, añazeas, añejo, añir, añacea, añojo, aragón, arcadus y alcadus, argamasa, armella, armiño, arriba, arte, atocha, avarraz, azagaya, azcona, azeytuna, azulejos // balumba, banca, baño, barbecho, barro, barbasco, basta, bastida, batiente, bausán, baza, bellón, banavar, benavente, berlenguas, besos, bexin, bisnaga, bibarrambla, brezo, breña, briviesca, brozno, brusco, brixiula, burbuja, burujo // caçon, ca̧̧orla, callo, cambio, camino, canasta, candamo, cantueso, carnero, carpintero, carta, cascabel, cavar, cojón, colcha, conciencia, conduchu,

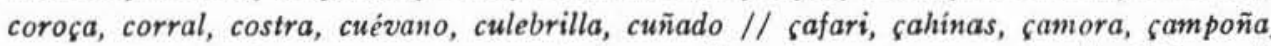
sanca, capata, cebratana, ceguta, cernicalo, cerrión, cisco, curana, susio // chipiona // de, de aqui adelante, demanda, dentón, devieso // écija, embarbascar, emelga, entredicho, enjalma, enxullo, epilepsia, era, ermar, escaña, escorzar, escudo, esparto, estrivo, estropeçar, exea // facinoroso, florentín, fraile // gafetí, galavardo, gallina, garça, garço, gárgola, garlito, garrapata, garrocha, gavanço, gasapo, gobi, goxe, guadalimar, gurbión, gusano // hienda, higuera de indias, hoja // jasar, jusbarra, illescas // librixa, librero, loba, lóbrego // madexa, mampesada, marhojo, mastranto, mastrates, meaja, melcocha, mella, mendrugo, messana, mesto, moheda, momia, morcilla, mordaça, mosaico, mostrenco, mugrón // naçulas, nebrixa, neguilla, nenúfar, nuca, nucz // oropéndola // páxaro // quexigo, quilatar // raya, reportorio, rezma // salvados, sarcia, saxifragua, sima, sirga, sisar, sobornal, socarrén, sonajas, sopetón, sorver, sorce, sosa, sovina, sulconete // tabique, tala, tamarindos, tarántula, tarreñas, terciopelo, teso, tilla, tiña, tiseras, tomar, tornadiço, trasdoblar, trença, trocatinte, trujamán, turbit, turnio, turón // xeme.

Esta lista de entradas permite afirmar que es Nebrija una de las autoridades más citadas por Covarrubias, aunque habría que hacer un cómputo detallado del número de entradas en que es citado el resto de las autoridades. Para las fuentes medievales dicho recuento ha sido realizado, en parte, por José Romera ${ }^{20}$, quien ofrece unos datos que, si los confrontamos con las 241 entradas presentadas en que se cita al sevillano, muestran el respeto que le tenía el autor del Tesoro.

\footnotetext{
"Autoridades literarias en el 'Tesoro' de Covarrubias", en Homenaje a Pedro Sainz Rodrigues, II (Estudios de Lengua y Literatura), Madrid, 1986, págs. 609-622.

10 Véase Manuel Seco, "Un lexicógrafo de la generación de Cervantes...", ya citado, pág. 238.

20 José Romera Castillo, “Don Juan Manuel...”, ya citado, pág. 314 y pág. 324. Véase también el estudio preliminar de la edición del Tesoro de la lengua castellana $o$ española preparada por Martín de Riquer, ya citada, págs. IX-X.
} 
A Nebrija recurre Covarrubias, sobre todo, para explicar las etimologias, pero también para definir las entradas, para indicar alguna variante, para citar textualmente una familia léxica entera o para indicar que Nebrija registra más variedades (véase, por ejemplo, azeytuna, hoja, librero) y, también, porque Nebrija registra la voz y Covarrubias se ve en la obligación de darle entrada (véase, por ejemplo, demanda, dentón, emelga). Al remitir Covarrubias a Nebrija, normalmente, utiliza las fórmulas "buelve", "según", "dize", "vide", "llama", "pone" Antonio Nebrixa, Antonio Nebrisense, Antonio de Nebrija, Antonio Nebrija, Antonio, etc.

No debemos olvidar que la intención de Covarrubias es "componer un diccionario etimológico, emulando con él en la lengua española lo que para la latina había hecho San Isidoro" ${ }^{21}$, coronando así "una tradición no muy larga de etimologistas del español, iniciada en 1565 por Alejo de Venegas, con la Declaración de algunos vocablos puesta como apéndice en su Agonía del tránsito de la muerte, y seguida por las Etimologías españolas (c. 1570) atribuidas al Brocense; la Recopilación de algunos nombres arábigos (1593), de Diego de Guadix; el Vocabulario etimológico (1600), de Bartolomé Valverde; el Origen y etymología de todos los vocablos originales de la lengua castellana (1601), de Francisco del Rosal, y Del origen y principio de la lengua castellana (1606), de Bernardo de Aldrete" ${ }^{22}$.

Covarrubias no anduvo muy acertado en la elección de Nebrija para las etimologías, ya que en la obra de Nebrija, como ha señalado Manuel Alvar Ezquerra ${ }^{23}$, más que de etimologías hay que hablar de correspondencias latinas de las voces. Sorprende, desde luego, la autoridad de Nebrija, latino ${ }^{24}$, cuando Covarrubias hace un diccionario etimológico para explicar que las voces vienen del hebreo, según una de las corrientes del siglo xvir. Claro que, como ha afirmado Martín de Riquer, "a nosotros no nos interesan primordialmente ni las etimologías - a pesar de su valor para el estudio de la evolución de la historia de la lengua - ni las antigüedades - aunque en éstas más de una vez nos explicamos claramente posiciones literarias de su tiempo, sobre todo por lo que se refiere a la fábula poética-; acudimos al Tesoro por su extraordinaria riqueza idiomática en voces, frases, dichos populares, refranes y por sus múltiples referencias a casos y hechos de su época, todo

21 Manuel Seco, "Un lexicógrafo de la generación de Cervantes ...", ya citado, pág. 231.

22 Ibídem, pág. 231.

23 Manuel Alvar Ezquerra, "Diccionario y gramática”, en LEA, IV, 1982, páginas 151-212, especialmente pág. 182; y Manuel Alvar Ezquerra, "Los prólogos del Diccionario académico: nomenclatura específica y microestructura", en RFE, LXIII, 1983, págs. 205-222, especialmente págs. 214-215.

24 Véase al respecto Manuel Seco, "Autoridades literarias...", ya citado, páginas 613-616. 
lo cual hace tiempo que se viene explotando para comentar y explicar nuestros mejores clásicos" ${ }^{25}$.

En definitiva, podemos afirmar que hay una clara presencia de Nebrija en Covarrubias y no sólo porque lo cite 243 veces, máxime si no es precisamente el empleo de autoridades lo más característico de este diccionario ${ }^{26}$, sino por las voces que toma, como veremos en seguida, sin citarlo. Es lo que suele hacer cuando dispone de suficiente información, como en el caso de canoa, voz documentada por primera vez en el Vocabulario de Nebrija, donde ni siquiera hace referencia a Antonio.

Debemos tener en cuenta, sin embargo, que no se puede hablar, en modo alguno, de plagio - la obra de Nebrija es bilingüe y, por tanto, no se extiende en informaciones prolijas, se limita al dato objetivo ${ }^{27}$, da la entrada más o menos especificada y su equivalencia latina; la de Covarrubias, en cambio, es una obra de carácter enciclopédico ${ }^{28}$-, sino de influencia. De la misma manera que reconoce haber tomado voces de Nebrija pudo tomar otras muchas como base y guia, y que luego amplió con informaciones muy heterogéneas. Dicha amplitud puede ser la causa de que la obra de Covarrubias - divaga siempre que le apetece- conste, según el recuento de Seco, de "16.929 (cifra que no corresponde a la de entradas, ya que con frecuencia una de éstas incluye, con definiciones, una familia léxica)" ${ }^{20}$; y la de Nebrija, en cambio, sea de 28.000 en el Diccionario ${ }^{30}$ y 22.500 en el Vocabulario $^{31}$.

Para comprobar la posible influencia de Nebrija en Covarrubias pretendemos realizar una comparación del léxico atesorado en la letra $A$ de la primera edición del Vocabulario de Nebrija y el de la letra $A$ del Tesoro de Covarrubias. Sería difícil averiguar qué edición o ediciones nebrisenses manejó Covarrubias en el momento de la creación de su diccionario y de ahí que hayamos preferido recurrir a la primera, pues no sólo es, evidentemente, la más antigua, sino también en la que ha habido menor posibilidad de en-

2.5 Martín de Riquer, estudio citado, pág. Ix.

26 Véase Manuel Seco, "Un lexicógrafo de la generación de Cervantes...", ya citado, pág. 237 y nota 25. Véase también Manuel Seco, “Autoridades literarias...”, ya citado, págs. 609-622.

27 Véase Manuel Seco, "Un lexicógrafo de la generación de Cervantes..., ya citado, pág. 235.

28 Véase Manuel Seco, ibidem, pág. 237, y Martín de Riquer, estudio citado, página virI.

29 Manuel Seco, "Un lexicógrafo de la generación de Cervantes...", ya citado, pág. 242 (nota 42 ).

so Elio Antonio de Nebrija, Diccionario latino-español. Salamanca (1492), estudio preliminar por Germán Colón y Amadeu-J. Soberanas, facsímil, Barcelona, PuvillEditor, 1979.

31 Véase Germán Colón y Amadeu-J. Soberanas, estudio citado, pág. 12 (nota 4). 
trecruzamiento, mezclas, presencia de elementos espurios, etc. No descartamos con ello que Covarrubias pueda haber utilizado otras ediciones; es más, estamos seguros de que así ha sido, ya que algunas de las voces en las que cita a Nebrija como autoridad no se hallan en la primera edición del Vocabulario. $\mathrm{Y}$ tampoco ciertas equivalencias y variantes que Covarrubias ofrece - pueden ser error del propio Covarrubias-; véanse, por ejemplo, salvados y reportorio.

Ofrecemos, como ejemplo de la mencionada influencia de Nebrija en Covarrubias, una muestra - el diccionario de Covarrubias hasta la $C$ es muy extenso- que abarca, siguiendo el orden alfabético presentado por Nebrija - mucho más coherente que el de Covarrubias, pues éste llega a escribir una misma entrada dos veces-, descle la entrada Almea ierva hasta Almivar ${ }^{32}$.

Almea ierva, cynoglo[โa. c.

Almea efta melma. lingua canina. Almeja pef́cado de concha. conchile. is.

Almena de torre o muro. pinna. $\varsigma$.

Almenara de huegos. ignes nocturni.

Almenara de açofar. lucerna polymyxos.

Almendro arbol conocida. amygdalus. i.

Almendra fruta del. amygdalum. i.

Almendra en latin, nux longa.

Almendral lugar dellos. amygdaletum. i.

Almendrada. amygdalinus cremor.

Almeria ciudad de efpaña. portus magnus.
Almea, "cierto genero de goma", "Almea, legun Antonio Nebril. por otro nombre azumbar".

Almeja, "vna elpecie de concha", "Ant. Neb. buelue, faba Aegyptia".

Almeida.

Almena, "fon las almenas to mas alto de los muros, a modo de torrezillas".

Almenara, "el fuego que fe haze en las torres de la colta, para dar auilo. Anton. Ignis Noturnos", "Tambien llaman a menaras. Anton. Neb. polymyxos".

$+++$

(Almendra), "fu arbol dicho Almendro". Almendra, "fruta conocida".

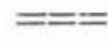

...........

Almeria, "ciudad aflentada a la ribera del Mar Mediterraneo".

Almete,

Almetoli.

32 Colocamos a la izquierda la entrada tal como se encuentra en el Vocabulario español-latino de Nebrija. A la derecha las entradas del Tesoro de la lengua castellana o española de Covarrubias. El doble subrayado sirve para señalar que se repite alguna entrada, de forma que mientras uno ofrece dos o tres, el otro sólo presenta una, lo cual no quiere decir que la entrada no haya sido recogida. La serie de cruces se emplea para indicar entradas que si bien no se recogen de manera exacta, pueden estar incluidas en la voz anterior - normalmente ésta presenta un carácter totalizador o genérico que engloba las distintas matizaciones de uno u otro autor-. Y la línea de puntos significa que la entrada falta en Nebrija o en Covarrubias. 


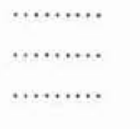

Almirez. aereum mortarium.

Almirante dela mar. nauarchus. i.

Almirante elte melmo, architalaflus, i. Almirantadgo. nauarchia. ę.

Almiralle en aravigo. interpretatur rex.

Almiron o cicorea. intubus, i.

Almivar. conditura ex faccaro.
Almez.

Almiar.

Almidon.

Almilla.

Almirez, "es el mortero de metal", "Anto. Neb. le llama aereum mortarium, liue abenum".

Almirante, "titulo grande en Elpaña, en Francia, y en Inglaterra".

\section{$==$}

(Almirante), "vale tanto como Principe. Ant. Neb. Almiralle en Arabigo interpretatur Rex".

Almiron, "vna elpecie de chicorea, Lat. intabus, vel intybus. Ant. Nebril. es nombre aravigo".

Almivar, "es çumo del membrillo, o de otra fruta incorporado con el açucar".

En esta comparación se observan claramente tres fenómenos:

1. Incorporación de los vocablos tal cual se encuentran en Nebrija, llegando a citar la información, como ya hemos comentado, al propio $\mathrm{Ne}-$ brija. Toma principalmente de Nebrija la etimología, algo que parece preocuparle tanto que le hace, algunas veces, olvidarse del significado.

2. Supresión de formaciones femeninas, derivados y gran parte de las matizaciones que Nebrija hacía en el Vocabulario, ya que en Covarrubias aparece como entrada sólo el lema base y es en su información interior donde figuran las distintas especificaciones que Nebrija presentaba en la propia entrada.

3. ${ }^{\circ}$ Adición de una serie de vocablos, algunos de los cuales estaban ya en Oudin, que sirvieron de fuente a Covarrubias. También hay que considerar las adiciones de entradas, si bien no de vocablos, cuando Covarrubias parece olvidarse de que determinados vocablos los había puesto antes.

Es representativo, pues, que de un total de 1.442 entradas contadas en la $A$ de Covarrubias —en la $A$ de Nebrija el número de entradas es de 2.437 -, 711 vocablos, que suponen 738 entradas, el $51,18 \%$, sean comunes; otros 22 vocablos, que suponen 24 entradas, el $1,67 \%$, se encuentren recogidas tanto en la obra de Nebrija como en la de Covarrubias, aunque la correspondencia no es totalmente exacta, ya que mientras uno de los autores presenta una sola entrada el otro ofrece dos o tres; 659 vocablos, que supo- 
nen 676 entradas, el 46,88\%, sean no comunes - en estas voces Covarrubias se muestra independiente de Nebrija-; y 4 vocablos, equivalentes a 4 entradas, el $0,27 \%$, no pertenezcan a la $A$-Covarrubias las registra en la $A \sin$ darse cuenta-

Por otra parte, incluso la idea del diccionario de Covarrubias tiene como trasfondo la misma preocupación renacentista existente en Nebrija: la defensa e ilustración de las lenguas nacionales ${ }^{33}$, que aparece de manera explícita en la dedicatoria de Covarrubias a Felipe III, donde dice: "de éste no sólo gozará la española, pero también todas las demás, que con tanta codicia procuran deprender nuestra lengua, pudiéndola agora saber de rayz, desengañados de que no se deve contar entre las bárbaras, sino ygualarla con la latina y la griega, y confessar ser muy parecida a la hebrea en sus frasis

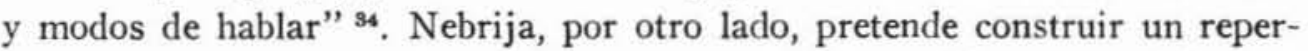
torio en el que no aparezcan elementos extranjeros y bárbaros, es decir, ajenos al latin con el que pretendía equiparar el castellano, al estar inmerso en esa corriente de culto latinizante que caracteriza el siglo $\mathrm{xv}$, siglo marcado por el sello latino, como han puesto de manifiesto Alvar y Mariner, Buceta y Fernández-Sevilla ${ }^{35}$.

Nebrija llega a afirmar que es necesario enmendar los manuscritos, recurriendo siempre que haya dudas a la lengua precedente: en latín al griego y en griego al hebreo ${ }^{30}$. Covarrubias, mordaz como Nebrija, afirma en el cuerpo del diccionario que no escribe para romancistas y presupone, por tanto, que sus lectores deben saber latín ${ }^{37}$; véanse, por ejemplo, las entradas abril, celoso, sátira.

Covarrubias, como prácticamente todos los lexicógrafos posteriores a Nebrija, ha sabido tener en cuenta una obra cumbre de la lexicografía española, sin la cual se habría perdido una gran riqueza léxica y cuya influencia llega hasta nuestros dias.

ss Véase Manuel Seco, "Un lexicógrafo de la generación de Cervantes...", ya citado, págs. 233-234.

34 Sebastián de Covarrubias, op. cit., fol. H. v.

35 Manuel Alvar y Sebastián Mariner, "Latinismos", en Enciclopedia Lingǘstica Hispánica, II (Elementos constitutivos. Fuentes, Madrid, C. S. I. C., 1967, págs. 3-49; Erasmo Buceta, "La tendencia a identificar el español con el latin. Un episodio cuatrocentista", en Homenaje ofrecido a Menéndes Pidal. Miscolánea de estudios linguiisticos, literarios e históricos, I, Madrid, Hernando, 1925, págs. 85-108; y Julio FernándezSevilla, "Un maestro preterido: Elio Antonio de Nebrija", en Thesaurus, XXIX, Bogotá, 1974, págs. 3-35.

36 Véase nuestra comunicación "Actitud de Nebrija ante los arabismos y mozarabismos", en Actas del I Congreso Internacional de Historia de la Lengua Española, I, págs. 873-880.

37 Véase Martín de Riquer, estudio citado, pág. virI, y Manuel Seco, "Un lexicógrafo de la generación de Cervantes ...", ya citado, pág. 235. 